\title{
ZFAS
}

\section{Vorwort von Bundesaußenminister Dr. Frank-Walter Steinmeier}

\author{
Frank-Walter Steinmeier
}

(C) Springer Fachmedien Wiesbaden 2014

Als ich im Dezember 2013 bei meiner Rückkehr in das Auswärtige Amt einen offenen Diskussionsprozess über Deutschlands Rolle in der Welt, über Maß und Grenzen unserer Verantwortung und über Ziele und Instrumente unserer Außenpolitik angestoßen habe, da wusste ich noch nicht, wie schnell die Frage nach der außenpolitischen Verantwortung unseres Landes sehr akut und sehr konkret werden würde.

In diesen Monaten überschlagen sich die Krisen: In der Ukraine, im israelischpalästinensischen Konflikt, in Syrien und Irak, mit der weltweiten Bedrohung durch das Ebolavirus. Dass sich an dieser beispiellosen Krisendichte in naher Zukunft etwas ändern wird, ist leider nicht abzusehen. Wir müssen im Gegenteil damit rechnen, dass die Krise der neue Normalfall ist. Hinzu kommt: Die Veränderungsgeschwindigkeit unserer Welt wird eher noch zunehmen als dass sie abnehmen wird. Kurzum: Die Welt scheint aus den Fugen geraten zu sein.

In dieser Situation könnte man versucht sein zu sagen: Jetzt ist die Zeit zum Handeln, nicht für Debatten. In der Tat sind wir mit dem Management der vielen Krisen sehr stark gefordert.

Warum halte ich diese Debatte trotzdem für nötig? Weil ich glaube, dass hinter der Häufung der Krisen tiefere Ursachen stehen. Wenn wir diesen nicht nachspüren, verpassen wir die Chance, künftig besser agieren und reagieren zu können. Ich habe den Eindruck, dass nicht nur die Welt eine neue Ordnung noch nicht gefunden hat. Sondern dass auch Deutschland noch nicht so recht verortet ist in dieser neuen, veränderten Welt.

\section{„Was ist falsch an der deutschen Außenpolitik?“ und: „Was sollte daran geändert werden?"}

Diese beiden Fragen habe ich zu Beginn des Projekts „Review 2014 - Außenpolitik Weiter Denken“ einer ganzen Reihe von Fachleuten aus dem In- und Ausland gestellt. 
Die Antworten sind hochinteressant und so weitreichend wie widersprüchlich: So fordern beispielsweise einige der Experten, Deutschland solle wieder die „Rolle des Mustereuropäers erlangen“, damit „,die Europäische Union revitalisieren“ und „als anerkannte Führungsmacht durch Europa den Lauf der Weltgeschichte verändern“. Gleichzeitig sollen wir eine ,präventive Stabilisierungspolitik in der europäischen Peripherie“ vorantreiben. Deutschland solle „interkultureller Vermittler" sein sowie eine „Brücke“ zwischen dem reichen Norden und dem ,aufstrebenden Süden“. Es solle „Russland europäisieren“ und „Amerika multilateralisieren“.

Eine Reihe von Autoren hat auch meine Einladung zu offener Kritik angenommen: „Deutschland reagiert eher auf Krisen, als seine Rolle in der Welt eindeutig wahrzunehmen und auszugestalten“" schreibt einer. Wir seien zwar ,in der Welt beliebt, aber ohne Karte und Kurs“. Strategisches Denken sei in Berlin „schwach ausgeprägt“. Und wir seien einerseits „,skrupellos realpolitisch“, andererseits „,naiv und idealistisch".

Die Lektüre dieser Außensichten ${ }^{1}$ zeigt: Die Erwartungen an unser Land unterscheiden sich je nach Perspektive deutlich voneinander. Aber sie haben einen gemeinsamen Nenner: Sie sind sehr hoch.

Dies kontrastiert auf den ersten Blick mit einer spürbaren Zurückhaltung der Menschen in Deutschland, wenn es um ein größeres deutsches Engagement in der Außenpolitik geht. Dieser Befund spiegelt sich in einer Umfrage der Körber-Stiftung, welche die Deutschen im Frühjahr dieses Jahres über ihre Sicht auf die Außenpolitik befragt hat: 37\% der Befragten wünschen sich mehr Verantwortung, also mehr Einmischung Deutschlands bei internationalen Krisen und Konflikten. Aber der größere Teil, nämlich $60 \%$ der Befragten, spricht sich dagegen aus.

Diese Skepsis erlebe ich in vielen Gesprächen und Diskussionen mit Bürgerinnen und Bürgern. Bei öffentlichen Debatten im Rahmen von „Review 2014 - Außenpolitik Weiter Denken“ äußert sich dies ein ums andere Mal, und zwar vor allem, wenn es um militärisches Eingreifen oder Waffenlieferungen geht.

Heißt das, die Deutschen scheuen internationale Verantwortung? Ich glaube nicht. Wenn man genauer hinschaut und hinhört, dann offenbaren sowohl die Umfrage als auch die vielen Debatten, die wir in den vergangenen Monaten geführt haben, dass unsere Bürger auch sehr hohe Erwartungen haben. Sie wollen deutsches Engagement, aber nicht so sehr militärisches. Sie wollen die Instrumente sehen, mit denen wir Krisen früh erkennen und vermeiden, sie managen und lösen. Sie wollen sehen, dass wir diplomatisch vermitteln, wo immer das möglich ist. Sie wollen, dass wir humanitär eingreifen, wo Menschen Not leiden. Und sie streiten auch untereinander über den richtigen außenpolitischen Weg: Nicht nur über Parteigrenzen, sondern auch zwischen den Generationen gibt es ganz unterschiedliche Perspektiven.

\section{Mehr außenpolitische Verantwortung wagen!}

Bei meinem zweiten Amtsantritt habe ich die These in den Raum gestellt: Deutschland ist ein bisschen zu groß und wirtschaftlich zu stark, um Außenpolitik nur von

${ }^{1}$ Alle Beiträge sind nachzulesen auf www.review2014.de. 
der Seitenlinie zu kommentieren. Wir müssen mehr außenpolitische Verantwortung wagen.

„Mehr Verantwortung“ - das ist weder der Ruf nach militärischen Abenteuern noch bedeutet es eine Abkehr von bewährten Grundprinzipien unserer Außenpolitik. Verantwortung ist immer konkret.

In Diskussionen über die Rolle unseres Landes höre ich immer wieder das Argument: „Mehr Verantwortung“ verbräme nur, das es uns in Wahrheit um Interessenpolitik gehe. Daran stimmt: Die Definition unser Interessen ist ein Teil der Antwort auf die Frage, wann, wo und wie wir ,mehr Verantwortung“ übernehmen müssen. Aber die zweite Frage, die wir uns meiner Meinung nach stellen müssen, ist: Wo können wir als Deutsche tatsächlich einen Unterschied machen?

Was wir brauchen, sind der Mut und die Bereitschaft, uns einzubringen. Die evangelische Theologin Dorothee Sölle hat einmal formuliert: „Wir dürfen uns nicht von der Ohnmacht überwältigen lassen. ,Da kann man nichts machen“ ist ein gottloser Satz".

Deshalb: Selbst in festgefahrenen Konflikten kann Außenpolitik etwas bewegen. Mit Augenmaß, Geduld, Gradlinigkeit und dem unbeirrbaren Willen zu verhandeln und zu versuchen, die Welt auch mit den Augen des anderen wahrzunehmen. Und in dem Wissen, dass es immer Alternativen zum Krieg gibt.

Den Begriff „mehr Verantwortung“ mit Leben zu erfüllen - das ist meine Aufgabe als Außenminister. Es ist aber zugleich eine Herausforderung für die wissenschaftliche und zivilgesellschaftliche Debatte in diesem Land: Tun wir das Richtige? Und tun wir es auf die richtige Art und Weise?

Eine solche außenpolitische Neu-Verortung wird nicht ohne Widersprüche zu erreichen sein. Sie wird niemals statisch sein. Und das darf sie auch nicht. Sondern die Herausforderung wird immer darin bestehen, in der konkreten Situation die richtige Antwort zu finden auf die Frage nach den Interessen unseres Landes und nach unseren Möglichkeiten, den Gang der Dinge zu beeinflussen. Das gilt kurzfristig für den richtigen Umgang mit drängenden Krisen. Das gilt aber auch langfristig für die Frage, wo und wie wir am besten zum Aufbau und zum Erhalt von Strukturen internationaler Ordnung beitragen können, von deren Nachhaltigkeit die Sicherheit und der Wohlstand unseres Landes ebenso wie der Frieden in der Welt sehr unmittelbar abhängen.

Ich bin überzeugt: Diese Debatte ist notwendig. Denn die Außenpolitik hat keine Wahl - sie muss versuchen, im Spannungsfeld zwischen einer sich dramatisch verändernden Welt, den hohen Erwartungen des Auslands an unsere Leistungsfähigkeit und den hohen Erwartungen des Inlands an unsere Kreativität einen vernünftigen, realistischen, wo immer möglich gradlinigen Weg zu finden. Denn nur so kann sie auf den notwendigen festen Grund bauen.

Die Autorinnen und Autoren dieses Sonderhefts leisten mit ihren Überlegungen, ihren Anregungen und auch mit ihren Erfahrungen aus der Praxis einen wertvollen Beitrag zu dieser Neu-Verortung. Ich empfehle es sehr gerne zur Lektüre. 